\title{
Recurrent Ureter Urothelial Carcinoma
}

National Cancer Institute

\section{Source}

National Cancer Institute. Recurrent Ureter Urothelial Carcinoma. NCI Thesaurus. Code C150516.

Reemergence of ureter urothelial carcinoma after a period of remission. 